\title{
Respostas funcionais foliares de plantas jovens de Hevea brasiliensis submetidas à deficiência hídrica e à reidratação
}

\author{
Functional leaf responses of young Hevea brasiliensis plants subjected to water \\ deficiency and rewatering
}

\section{Nayara Fonseca do NascimentoI, Larissa Batista de Brito do Nascimento ${ }^{\text {II }}$, José Francisco de Carvalho Gonçalves ${ }^{\text {III }}$}

\begin{abstract}
Resumo
A deficiência hídrica é considerada um fator que produz danos significativos durante o período de estabelecimento e de crescimento dos plantios. O objetivo deste estudo foi investigar características morfofisiológicas e bioquímicas de folhas de mudas de seringueira em resposta à deficiência hídrica e, posterior, reidratação. Plantas jovens de Hevea brasiliensis do clone RRIM 600 - Rubber Research Institute of Malaysia foram divididas em dois tratamentos, tratamento 1 = plantas irrigadas (IR) e tratamento 2 = plantas não irrigadas (NI). Nestas plantas, a fotossíntese $(A)$ foi acompanhada durante todo o período experimental $(0$, 10, 25, 32, 38, 46, 54 e 68 dias). O potencial hídrico foliar ( $\left.\psi_{w}\right)$ foi determinado usando-se uma câmara de pressão do tipo Scholander, a fotossíntese $(A)$, condutância estomática $(g s)$ e transpiração $(E)$ foram determinadas usando-se um analisador de gás infravermelho (LI-6400XT, LI-COR, Lincoln, Nebraska, USA) e o conteúdo de carboidratos foliares e de prolina foram determinados por espectrofotometria (Amersham Biosciences Ultrospec 3100 pro). O $\psi_{w}, A$, gs, $E$ e o conteúdo de amido foliar no tratamento NI foram reduzidos durante os primeiros 32 dias, em que $A$ atingiu valores próximos a $0 \mu \mathrm{mol} \mathrm{m}^{-2} \mathrm{~s}^{-1}$. Porém, houve aumento da concentração de açúcar solúvel total e de prolina (tratamento NI) neste mesmo período. A recuperação da fotossíntese (NI) ocorreu após 22 dias depois da reidratação, quando comparado ao tratamento (IR). Portanto, pode-se concluir que o clone RRIM 600 possui tolerância ao estresse hídrico em termos de como lidar com o estresse hídrico progressivo, mas a recuperação ao estresse foi relativamente lenta.
\end{abstract}

Palavras-chave: Carboidratos; Crescimento; Estresse; Trocas gasosas

\begin{abstract}
Hydric deficit is considered a factor that produces significant damage during the establishment and growth period of plantations. The goal of this study was to investigate morphophysiological and biochemical traits of leaves of the rubber seedlings in response to water deficiency and subsequent rewatering. Young Hevea brasiliensis plants of the RRIM 600 clone - Rubber Research Institute of Malaysia were divided into two treatments: treatment $1=$ irrigated plants (IR) and treatment $2=$ non-irrigated plants (NI). In these plants the photosynthesis $(A)$ was monitored throughout the experimental period $(0,10,25,32,38,46,54$ and 68 days). The water potential in leaves $\left(\psi_{\mathrm{w}}\right)$ was determined using a Scholander pressure chamber. The photosynthesis $(A)$, conductance $(g s)$, transpiration $(E)$ were determined using an infrared gas analyzer (LI6400XT, LI-COR, Lincoln, Nebraska, USA) and leaf carbohydrates and proline content were determined by spectrophotometry (Amersham Biosciences Ultrospec 3100 pro). The $\psi_{\mathrm{w}}, A, g s, E$ and leaf starch content in treatment NI were reduced during the first 32 days, where $A$ reached value close to $0 \mu \mathrm{mol} \mathrm{m}^{-2} \mathrm{~s}^{-1}$. However, there was an increase in the concentration of total soluble sugar and proline during the same period. The recovery of photosynthesis (NI) occurred at 22 days after the rewatering when compared to irrigated plants (IR). Therefore, it can be concluded that the RRIM 600 clone has water stress tolerance in terms of how to deal with the progressive water stress, but the recovery from stress was relatively slow.
\end{abstract}

Keywords: Carbohydrates; Growth; Stress; Gas exchange

Engenheira Florestal, MSc., Doutoranda em Engenharia Florestal no Centro de Ciências Rurais, Universidade Federal de Santa Maria - UFSM, Av. Roraima, 1000, CEP 97105-900, Santa Maria (RS), Brasil. nayaraeng.florestal@gmail.com (ORCID: 0000-0002-3533-0451)

Engenheira de Bioprocessos, Mestranda em Biotecnologia na Universidade Federal do Amazonas - UFAM, Av. general Rodrigo Octávio, 6200, CEP 69080900, Manaus (AM), Brasil. lbbnascimento@gmail.com (ORCID: 0000-0001-6083-8399)

III Engenheiro Agrônomo, Dr., Pesquisador Titular III do Instituto Nacional de Pesquisas da Amazônia - INPA, Av. André Araújo, 2936, CEP 69067375, Manaus (AM), Brasil. jfc@inpa.gov.br (ORCID: 0000-0001-9197-4617) 


\section{Introdução}

A seringueira (Hevea brasiliensis Willd. Ex A. Juss. Müll. Arg) é uma árvore cujo centro de dispersão é a Região Amazônica e o seu principal produto é o látex, matéria-prima para produção da borracha. A despeito da origem amazônica, atualmente, os maiores produtores de borracha do mundo são os países orientais e asiáticos como a Tailândia, Indonésia, Malásia, Vietnã, Filipinas, Myanmar e Camboja (LI; FOX, 2012). Desde o início dos plantios, a seringueira passou por vários processos de seleção de clones que facilitaram a expansão dos cultivos para locais com climas distintos ao da Amazônia, encontrando algumas vezes climas mais favoráveis para a heivecultura, permitindo maior adaptabilidade e aumento na produção dos plantios (PRIYADARSHAN et al., 2005). Em termos de produção, ao analisar os resultados dos programas de melhoramento da seringueira pelo mundo chega-se à conclusão que o clone do Rubber Research Institute of Malaysia (RRIM 600) é muito produtivo e também um dos mais plantados globalmente (LAU et al., 2016).

A produtividade vegetal, em geral, é garantida pelo desempenho funcional das espécies, que por sua vez é influenciado pelo potencial genético e pelas condições bióticas e abióticas do ambiente de crescimento. Porém, independentemente da genética da espécie e do meio de crescimento, a produtividade quase sempre é comprometida por fatores estressantes. As alterações nos fatores abióticos afetam sobremaneira a atividade fisiológica das plantas em suas diferentes fases ou estádios de crescimento (ANJUM et al., 2011). Fatores estressantes como alta irradiância, deficiência nutricionais e hídricas tornam mais difícil o sucesso no estabelecimento das plantas na fase juvenil. Além disso, as condições estressantes individuais também podem ser potencializadas na medida em que, não raro, ocorrem sobreposições de diferentes estresses, ou seja, ambientes de alta irradiância quase sempre condicionam as plantas a sofrerem estresses térmico e hídrico e, o baixo fluxo de água na planta pode levar a deficiência nutricional (SANTOS JUNIOR; GONÇALVES; FELDPAUSCH, 2006).

Do ponto de vista global, tem sido bastante divulgado que os cenários futuros indicam a ocorrência de eventos extremos para aumento de temperatura e maiores períodos com baixas precipitações, além de maior frequência destes eventos, que se seguem como consequência das mudanças climáticas globais (JIMÉNEZ-MUÑOZ et al., 2016). Na literatura, também é possível verificar que a seca se caracteriza como o estresse ambiental mais significativo na Amazônia, expondo as plantas às condições estressantes acima mencionadas com maiores frequência e intensidade (JIMÉNEZ-MUÑOZ et al., 2016).

Em estudo com a Hevea brasiliensis foi demonstrado que, do ponto de vista funcional, a regulação estomática ocorre para manter a diferença entre o potencial hídrico das folhas e o potencial do solo constante no período de seca (KUMAGAI et al., 2015) e evitar a perda da função hidráulica (CHEN et al., 2010). Em plântulas de Hevea brasiliensis, o fechamento estomático ocorre devido a sinalizações químicas e hidráulicas, sendo realizado para tentar evitar a cavitação no xilema e a falha hidráulica (CHEN et al., 2010). No entanto, para a seringueira, ainda não está devidamente esclarecido como podem ocorrer alterações bioquímicas derivadas do estresse hídrico, em particular, aquelas que envolvem maiores acúmulos de substâncias osmoticamente ativas que participam da regulação hídrica nas células (WANG, 2014). Assim como, faltam informações sobre as alterações nos teores de pigmentos cloroplastídicos nas plantas de Hevea brasiliensis, que são moléculas fundamentais para a captura de luz no processo de fotossíntese e, como consequência, pode implicar estreita relação com o ganho de biomassa (ANJUM et al., 2011). Além disso, outras consequências indesejáveis provenientes da falta de água que diminuem a produtividade e têm reflexos diretos sobre o metabolismo do carbono e a produção de látex nas plantas adultas de seringueira ainda precisam ser mais bem compreendidas (KUNJET et al., 2013).

Estudos que evidenciem as modificações fisiológicas da espécie em diferentes condições de crescimento são fundamentais para entender os processos adaptativos que ocorrem, algumas vezes, precocemente e que podem acarretar grandes limitações no futuro para o desempenho das plantas sob condições de plantios. Adicionalmente, estas pesquisas somam informações cientificas para programas de melhoramento genético da espécie (GAONA et al., 2018). Neste 
contexto, estudar os aspectos morfofisiológicos que influenciam a capacidade de plantas jovens de Hevea brasiliensis à adaptação/aclimatação das plantas às situações de deficit hídrico e, posteriormente, a sua capacidade de recuperação no pós-estresse, por meio da reidratação representa uma abordagem experimental acurada para fornecer indicadores relacionados à plasticidade funcional da espécie/clones e aos desdobramentos práticos que as características funcionais podem revelar, neste caso, a necessidade ou não de irrigação e de ciclos de rega. Ademais, a compreensão das respostas morfofisiológicas e bioquímicas à seca torna-se essencial para uma percepção holística dos mecanismos de tolerância das árvores quando submetidas à condição de deficiência hídrica. O objetivo deste trabalho foi de investigar as características morfofisiológicas e bioquímicas de plantas jovens de Hevea brasiliensis em resposta à deficiência hídrica e a subsequente reidratação. Considerando o alto desempenho funcional do clone RRIM 600 , investigou-se a hipótese que este clone teria tolerância diferencial à deficiência hídrica e que os indicadores fisiológicos relacionados às respostas de tolerância ao estresse poderiam estar ligados à estabilidade do aparato fotossintético.

\section{Material e método}

\section{Material vegetal}

O experimento foi conduzido em área situada no Instituto Nacional de Pesquisas da

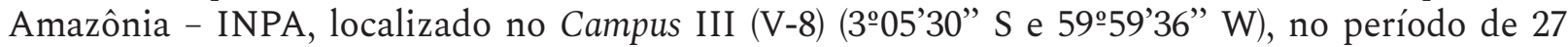
de novembro 2016 a 2 de fevereiro de 2017. Todas as análises fisiológicas e bioquímicas foram realizadas no Laboratório de Fisiologia e Bioquímica Vegetal (LFBV) do INPA.

Para a realização do experimento foram utilizadas plantas jovens (mudas) enxertadas com aproximadamente 7 meses de idade, do clone RRIM 600 (Hevea brasiliensis) provenientes da empresa Amazônia viva, localizada no município Rio Branco - Acre. No laboratório de Fisiologia e Bioquímica Vegetal - INPA, as plantas foram transferidas para vasos com capacidade para $10 \mathrm{~L}$, contendo mistura de terriço vegetal. Todas as plantas foram irrigadas até a capacidade de campo (aproximadamente $4 \mathrm{~L}$ de água) e aclimatadas durante 30 dias, em casa de vegetação de cobertura transparente para evitar incidência direta de luz e prover abrigo de chuva. Durante todo o período de aclimatação, as plantas receberam irrigação diária. A temperatura no ambiente interno à casa de vegetação foi de $31 \pm 2{ }^{\circ} \mathrm{C}$ durante a execução do experimento, no período do meio-dia.

\section{Condução do experimento}

Após a aclimatação (30 dias), as plantas foram separadas em dois grupos, caracterizando dois (2) tratamentos, o primeiro de plantas irrigadas (IR) e o segundo de plantas não irrigadas (NI), compostos por 10 indivíduos (mudas) por tratamento, todas as mudas foram padronizadas a priori em termos de altura, diâmetro e números de folhas. Após a aclimatação, as mudas foram submetidas às primeiras medições (condição aclimatada ou tempo 0). Após a primeira medição, a irrigação foi suspensa para o tratamento NI, até que as determinações da fotossíntese exibissem valores próximos a zero $\left(0 \mu \mathrm{mol} \mathrm{m} \mathrm{m}^{-2} \mathrm{~s}^{-1}\right)$. Quando isso aconteceu, este tratamento foi novamente irrigado até a capacidade de campo, e irrigado diariamente até que fosse recuperado o patamar fotossintético semelhante do tratamento IR. O parâmetro fotossíntese foi utilizado como indicador fisiológico, pois já foi demonstrado que é um dos primeiros parâmetros a ser afetado sob deficiência hídrica, e o último a ser recuperado (LIBERATO et al., 2006; GONÇALVES; SILVA; GUIMARÃES, 2009).

As avaliações de trocas gasosas e da fluorescência da clorofila $a$ foram realizadas durante todo o período experimental $(0,10,25,32,38,46,54$ e 68 dias). Por outro lado, as variáveis de área foliar, área foliar específica, potencial hídrico, pigmentos cloroplastídicos, índice de conteúdo de 
clorofila, açúcar solúvel, amido e prolina foram avaliados considerando-se três cenários, a saber: 1 - condição aclimatada; 2 - supressão da irrigação no tratamento não irrigado (NI) até as leituras de fotossíntese alcançar valores próximos de zero; 3 - reidratação em NI até o registro de ausência de diferenças significativas entre as leituras de fotossíntese realizadas em no tratamento IR e NI (GONÇALVES; SILVA; GUIMARÃES, 2009).

\section{Área foliar e área foliar especifica}

Para a medição da área foliar total foi utilizado scanner na resolução de 600 dpi e as imagens foram processadas no software IMAGE J. A área foliar específica (AFE) foi determinada a partir de dez discos foliares de área conhecida $\left(0,283 \mathrm{~cm}^{2}\right)$ retirados de cada planta, acondicionados em sacos de papel e submetidos à secagem em estufa a temperatura de $65^{\circ} \mathrm{C}$ durante 72 horas. A AFE foi obtida pela razão entre a área foliar $\left(\mathrm{cm}^{2}\right)$ e massa foliar seca (g) (EVANS; POORTER, 2001).

\section{Potencial hídrico foliar}

O potencial hídrico foliar foi determinado em dois diferentes horários, antemanhã (IPD) medido entre $6 \mathrm{~h}$ e $7 \mathrm{~h}$ e meio-dia ( $\Psi M D$ ) com medições realizadas entre $12 \mathrm{~h}$ e $13 \mathrm{~h}$, utilizandose uma câmara de pressão do modelo 3005-1422 (Soil Moisture Equipment Corp, USA) (SCHOLANDER et al., 1964). As medições foram realizadas nas plantas no interior da casa de vegetação. As amostras foliares de cinco plantas por tratamento foram coletadas e submetidas à pressão de $\mathrm{N}_{2}$ gasoso até ocorrer exsudação da seiva através do pecíolo foliar.

\section{Trocas gasosas foliares}

As determinações fotossintéticas foram realizadas em cinco plantas por tratamento, utilizando-se o folíolo central, localizados no terço-médio de cada planta, em folhas completamente expandidas e estado fitossanitário adequado. Sendo medidas as taxas de fotossíntese $(A)$, condutância estomática $(g s)$, transpiração $(E)$ e respiração no escuro $(\mathrm{Rd})$ realizadas com analisador de gás infravermelho portátil, de sistema aberto LI-COR 6400 (Li-Cor, Nebraska, USA).

As medições foram realizadas sempre no período entre $8 \mathrm{~h}$ e $12 \mathrm{~h}$. Os dados pontuais de trocas gasosas foram obtidos à densidade de fluxo de fótons saturante (PPFD) de $1000 \mu \mathrm{mol}$ $\mathrm{m}^{-2} \mathrm{~s}^{-1}$ (WANG, 2014). O equipamento foi ajustado para o fluxo de $400 \mu \mathrm{mol} \mathrm{s} \mathrm{s}^{-1}$, concentração de $\mathrm{CO}_{2}$ e $\mathrm{H}_{2} \mathrm{O}$ dentro da câmara de medição em torno de $400 \pm 4 \mu \mathrm{mol} \mathrm{mol}{ }^{-1}$ e $21 \pm 1 \mathrm{mmol}$ $\mathrm{mol}^{-1}$, respectivamente. A temperatura foi mantida em torno de $31 \pm 1^{\circ} \mathrm{C}$ (GONÇALVES; SILVA; GUIMARÃES, 2009).

\section{Fluorescência da clorofila $a$}

A determinação da fluorescência da clorofila $a$ foi realizada no folíolo central. O monitoramento da fluorescência da clorofila $a$ foi realizado em cinco plantas por tratamento, para as medições utilizou-se um fluorômetro portátil (Plant Efficiency Analyser - MK2 - 9600, Hansatech, Norfolk, UK). Todas as determinações sempre foram realizadas entre $8 \mathrm{~h}$ e $12 \mathrm{~h}$, em folhas completamente expandidas. Inicialmente, as folhas foram aclimatadas ao escuro durante 30 minutos e posteriormente, foram expostas a um pulso de luz saturante de $650 \mathrm{~nm}$ durante $1 \mathrm{~s}$, numa intensidade de $3000 \mu \mathrm{mol} \mathrm{m} \mathrm{m}^{-2} \mathrm{~s}^{-1}$. A partir das leituras foram obtidos o rendimento quântico máximo do PSII (Fv/Fm), o índice de desempenho (PIABS) (STRASSER et al., 2010). 


\section{Teores de pigmentos cloroplastídicos}

Os teores de pigmentos cloroplastídicos foram determinados a partir de $0,1 \mathrm{~g}$ de material vegetal fresco, proveniente de folhas que apresentavam estado fitossanitário considerado adequado e de cinco plantas por tratamento. Este material foi homogeneizado com $10 \mathrm{~mL}$ de acetona $(80 \% \mathrm{v} / \mathrm{v})$ e $\mathrm{MgCO}_{3}(0,5 \% \mathrm{p} / \mathrm{v})$ em banho de gelo e na ausência de luz. Posteriormente, o extrato foi filtrado em papel-filtro qualitativo $\mathrm{n}^{\circ} 2$. Depois de filtrado, o volume do extrato foi aferido para $15 \mathrm{~mL}$ com a mesma solução. O extrato foi lido em espectrofotômetro (Ultrospec 2100 pro UV/visible, Amersham, Biosciences, Cambridge, UK). As absorbâncias das clorofilas a (chla), clorofila $b$ (chlb) e carotenoides $(\mathrm{Cx}+\mathrm{c}$ ), utilizadas foram $\lambda=663,645$ e $480 \mathrm{~nm}$ (LICHTENTHALER; WELLBURN, 1983). As concentrações das clorofilas no extrato foram calculadas na base da massa foliar $\left(\mu \mathrm{mol} \mathrm{g}^{-1}\right)$, a partir das equações descritas por Hendry e Price (1993).

\section{Extração de carboidratos (açúcares solúveis totais e amido foliares)}

Para a determinação dos carboidratos, as folhas foram coletadas durante a manhã e depois de secas em estufa a $65^{\circ} \mathrm{C}$, até obtenção de massa constante. Em seguida, as folhas foram moídas até a granulometria de um pó fino, sendo utilizados para posterior análise. O amido foi extraído do precipitado restante dos açúcares solúveis total (AST) após a extração com $10 \mathrm{~mL}$ de ácido perclórico $35 \%(\mathrm{v} / \mathrm{v})$ e centrifugado a $10.000 \mathrm{rpm}$ durante 15 minutos, a $25^{\circ} \mathrm{C}$, recolhendose o sobrenadante. Os AST e amido das folhas foram determinados seguindo o método fenolsulfúrico a leitura espectrofotométrica (Ultrospec 2100 pro) foi feita na absorbância de $\lambda=490$ nm (DUBOIS et al., 1956). A estimativa de açúcares solúveis totais e amido foi feita utilizando-se equação ajustada com curva padrão estabelecida com glicose (Sigma).

\section{Prolina}

Os teores de prolina das folhas foram determinados utilizando-se a metodologia descrita por Bates, Waldren e Teare (1973). O material foi coletado pela manhã, em seguida o material fresco foi moído em almofariz usando nitrogênio líquido até obtenção de um pó fino. Cerca de $0,5 \mathrm{~g}$ da amostra foi homogeneizada com ácido sulfossalicílico $3 \%$ e o sobrenadante foi submetido à presença de ninidrina e o produto da reação lido em espectrofotômetro no comprimento de onda $\lambda=520 \mathrm{~nm}$. A concentração de prolina livre foi calculada a partir de equação ajustada utilizando prolina (Sigma) como um padrão.

\section{Desenho experimental e análises estatísticas}

O delineamento experimental utilizado foi o inteiramente casualizado (DIC), obedecendo experimento fatorial $2 \times 3$ (dois tratamentos hídricos, IR e NI, versus três períodos experimentais $0,32 \mathrm{e}$ 54 dias), representando os períodos das plantas aclimatadas, com deficit hídrico e durante a recuperação do estresse, respectivamente. Cada tratamento foi composto por 10 mudas. Todos os dados foram submetidos a testes para verificação do cumprimento das premissas de normalidade e homogeneidade de variâncias, respectivamente. Nos casos em que esta estratégia não foi suficiente para atendimento às premissas foram feitas transformações dos dados, quando não satisfeitas as premissas, aplicou-se o teste não paramétrico U de Mann-Whitney $(\mathrm{P}<0,05)$. Por outro lado, quando satisfeitas as premissas, utilizouse o teste paramétrico $t$ de Student para amostras independentes (tratamento irrigado x tratamento não irrigado) $(\mathrm{P}<0,05)$. Todas as análises foram realizadas utilizando-se o programa SIGMA PLOT 11.0. 


\section{Resultados e discussão}

\section{Morfologia}

No presente estudo não foram verificadas alterações significativas na área foliar de plantas jovens de Hevea brasiliensis em nenhum dos períodos avaliados (Tabela 1). No entanto, deve registrar-se que as plantas apresentaram, no tratamento NI, acelerado processo de senescência e de abscisão das folhas, havendo posterior lançamento de folhas novas. Nas folhas novas, a possibilidade de implementações de mudanças foliares, sejam estas de caráter morfológico e/ ou funcional, pode ser mais evidente do que nas folhas que iniciaram o experimento, mesmo que as folhas originais apresentassem diferentes estádios fenológicos. Embora seja relatado na literatura que a redução da área foliar de plantas sob estresse hídrico seja direcionada para reduzir a transpiração e proteger as plantas contra potenciais danos oxidativos devido à menor superfície de exposição à luz, estas alterações quase sempre implicam menor produção de biomassa foliar em plantas que apresentam deciduidade das suas folhas, como é o caso da seringueira (CHEN et al., 2010). Mas, o uso de clones mais tolerantes a diferentes tipos de estresses pode minimizar ou prolongar as respostas ecofuncionais das plantas, admitindo-se também a importância do tempo e da intensidade do estresse para verificação das alterações morfofuncionais.

\section{Tabela 1 - Morfologia foliar de plantas jovens de Hevea brasiliensis crescendo na condição aclimatada (0); deficit hídrico (32 dias) e recuperação (54 dias).}

Table 1 - Leaf morphology of young plants of Hevea brasiliensis growing in the acclimated condition (0); water deficit (32 days) and recovery (54 days).

\begin{tabular}{lcccc}
\hline \multirow{2}{*}{ Tempo (dias) } & \multicolumn{2}{c}{ AF $\left(\mathbf{c m}^{2}\right)^{*}$} & \multicolumn{2}{c}{$\mathbf{A F E}\left(\mathbf{c m}^{2} \cdot \mathbf{g}^{-1}\right)^{*}$} \\
\cline { 2 - 5 } & Irrigada & Não irrigada & Irrigada & Não irrigada \\
\hline $\mathbf{0}$ & $111,33 \pm 17,90(\mathrm{~A})$ & $122,12 \pm 37,45(\mathrm{~A})$ & $237,42 \pm 62,53(\mathrm{~A})$ & $241,71 \pm 64,49(\mathrm{~A})$ \\
$\mathbf{3 2}$ & $132,21 \pm 37,98(\mathrm{~A})$ & $125,46 \pm 28,16(\mathrm{~A})$ & $210,96 \pm 21,86(\mathrm{~A})$ & $181,07 \pm 7,48(\mathrm{~B})$ \\
$\mathbf{5 4}$ & $122,83 \pm 28,30(\mathrm{~A})$ & $118,32 \pm 39,39(\mathrm{~A})$ & $193,54 \pm 12,28(\mathrm{~A})$ & $205,03 \pm 35,02(\mathrm{~A})$ \\
\hline
\end{tabular}

Em que: $\mathrm{AF}=$ Área foliar. AFE $=$ Área foliar específica. ${ }^{*}=$ Valores médios e respectivos desvios padrões. Médias seguidas da mesma letra maiúscula entre os tratamentos não diferem entre si pelo teste $t(P<0,05)$, a partir da segunda coluna.

Em contrapartida, quando a área foliar especifica (AFE) foi analisada, aos 32 dias (sob deficiência hídrica, tratamento NI) verificou-se redução cerca de 14\% (Tabela 1). Porém, na avaliação final houve recuperação da AFE a valores semelhantes àqueles obtidos para o tratamento irrigado. A AFE representa a razão da superfície pela massa seca da folha, ou seja, se conceitualmente a superfície relaciona-se à parte morfológica e a fitomassa deve ser associada ao componente estrutural, como a composição interna, ao número e ao tamanho das células do mesofilo, pode-se inferir que houve aumento do mesofilo por unidade de área foliar. Sendo o aumento da espessura do parênquima paliçádico uma das respostas das plantas à deficiência hídrica (MELO et al., 2017). Logo, valores mais baixos de AFE significam folhas mais espessas, que podem estar associadas a mecanismos para aumentar a eficiência no uso da água, sugerindo maior número de células do mesofilo. 


\section{Potencial hídrico foliar}

O potencial hídrico $\left(\Psi_{\mathrm{w}}\right)$ das plantas jovens de Hevea brasiliensis, no tratamento NI aos 32 dias, foi reduzido no período das seis horas da manhã, sendo mais acentuado ao meio-dia (Figura 1 A e B).

Figura 1 - Potenciais hídricos foliares medidos a $6 \mathrm{~h}(\mathrm{~A})$ e $12 \mathrm{~h}(\mathrm{~B})$, em plantas jovens de Hevea brasiliensis.

Figure 1 - Leaf water potential measured at 6:00 a.m. (A) and 12:00 a.m. (B), in young plants of Hevea brasiliensis.

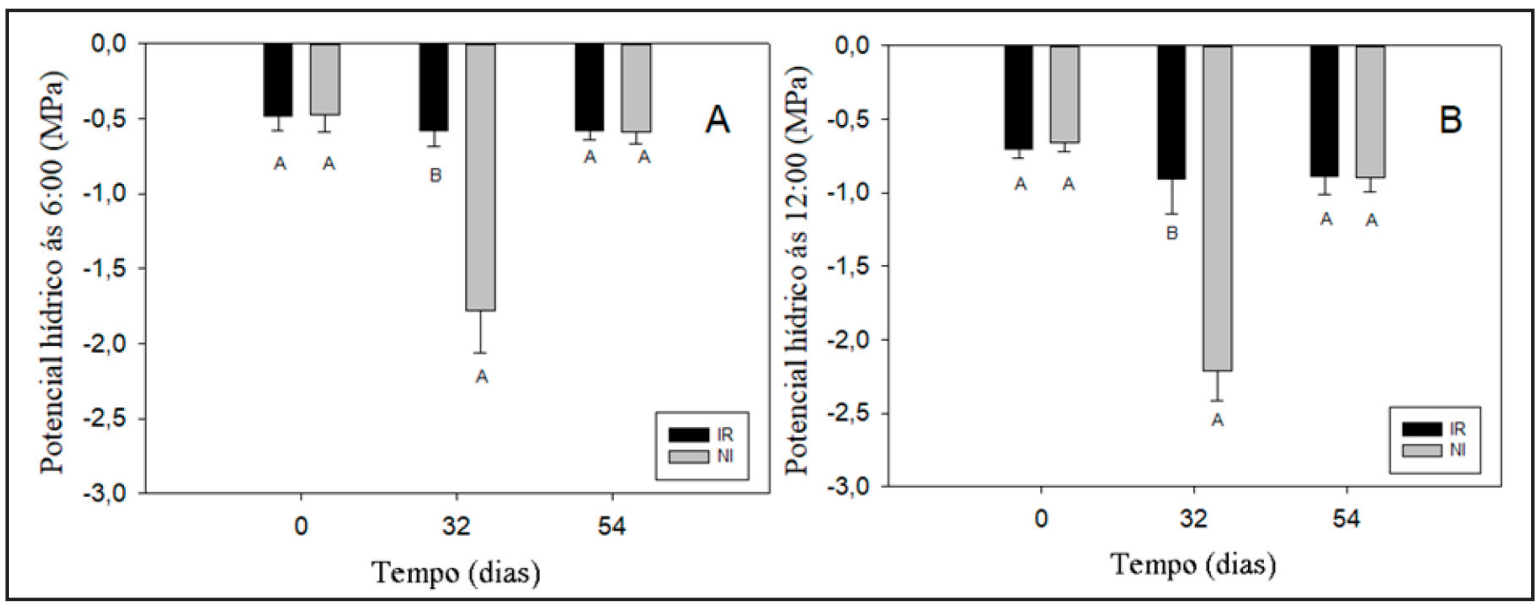

Fonte: Autores (2017)

Os valores são médias e as linhas verticais indicam os erros padrões. Letras iguais para cada período indica ausência de diferença significativa entre os tratamentos pelo teste $t(\mathrm{P}<0,05)$.

Values are averages and vertical lines indicate standard errors. Equal letters for each period indicate absence of significant difference between treatments by the test $\mathrm{t}(\mathrm{P}<0.05)$.

A redução do $\Psi_{\mathrm{w}}$ foliar durante o dia está relacionada ao aumento da taxa de transpiração, que ocorre devido à alta demanda evaporativa da atmosfera. Em estudo com Hevea brasiliensis sob restrição hídrica após 11 dias, o potencial atingiu -3,0 MPa (CHEN et al., 2010). Entretanto, no presente estudo, o menor potencial hídrico medido foi próximo de $-2,5 \mathrm{Mpa}$, encontrado somente aos 32 dias sem irrigação. Por outro lado, em estudo com vários clones de seringueira sob deficit hídrico foram encontrados valores similares ao registrado no presente estudo (JINAGOOL et al., 2015). De certa forma, estas variações no status hídrico foliar podem ser atribuídas às diferenças intraespecíficas e às condições reinantes de cada experimento. Quanto à reidratação, foi possível confirmar que após 32 dias, o tratamento NI já exibia valores estatisticamente semelhantes para o potencial hídrico, nos dois horários avaliados, quando comparado ao tratamento IR. Também com seringueira foi demonstrada uma recuperação bem mais rápida, entre 3 a 5 dias, as plantas sob estresse apresentam valores similares ao controle (CHEN et al., 2010). Essas diferenças de respostas funcionais podem estar relacionadas a diversos motivos, destacando-se as condições experimentais, o substrato de crescimento, as variáveis microclimáticas, os tipos de clones, etc., mas nesta comparação, especificamente, parece que a idade dos clones pode ter sido fator determinante para proporcionar as diferenças no potencial hídrico, no presente estudo as plantas de Hevea brasiliensis estavam com 7 meses e no trabalho de Chen et al. (2010) as plantas estavam com 2 anos, provavelmente, estas plantas apresentavam arquitetura hidráulica mais bem estruturada. 


\section{Trocas gasosas foliares}

As taxas fotossintéticas variaram de $0,4 \mu \mathrm{mol} \mathrm{m}^{-2} \mathrm{~s}^{-1}$ sob deficit hídrico (fotossíntese líquida próxima a zero) até $8,4 \mu \mathrm{mol} \mathrm{m} \mathrm{s}^{-1}$ nas plantas jovens de Hevea brasiliensis bem hidratadas. Estes valores representam expressiva faixa de variação para fotossíntese em aproximadamente 21 vezes, logo sugerese que a quantidade de água disponível influenciou significativamente a fotossíntese, visto que, este parâmetro foi o primeiro (10 dias após a supressão da irrigação), a se diferenciar do controle, seguido da gs e $E$ (Figura 2A). A redução da $A$ relaciona-se fortemente ao fechamento estomático, algo já bem conhecido na literatura (GONÇALVES; SILVA; GUIMARÃES, 2009). A regulação estomática ocorre para manter a diferença entre o potencial hídrico das folhas e o potencial do solo constante no período de seca (KUMAGAI et al., 2015). No entanto, o que chama a atenção é que esta redução da $A$, sob deficit hídrico, apresenta certas características inerentes ao dano no aparato fotossintético, a redução na síntese de ATP, a diminuição da atividade da ribulose-1,5-bisfosfato carboxilase/oxigenase ou mesmo decréscimos na taxa de regeneração desta moléculas que têm implicações sobre o metabolismo do carbono como um todo, inclusive na respiração (Figura 2B), a começar pela limitação estomática ao influxo de $\mathrm{CO}_{2}$ até chegar, supostamente, ao nível mitocondrial (LIBERATO et al., 2006; GONÇALVES; SILVA; GUIMARÃES, 2009).

Figura 2 - Fotossíntese (A); Respiração (B); Condutância estomática (C); transpiração (D) de plantas jovens de Hevea brasiliensis irrigadas e não irrigadas.

Figure 2 - Photosynthesis (A); Respiration (B); Stomatal conductance (C); transpiration (D) of young plants of Hevea brasiliensis irrigated and non-irrigated.

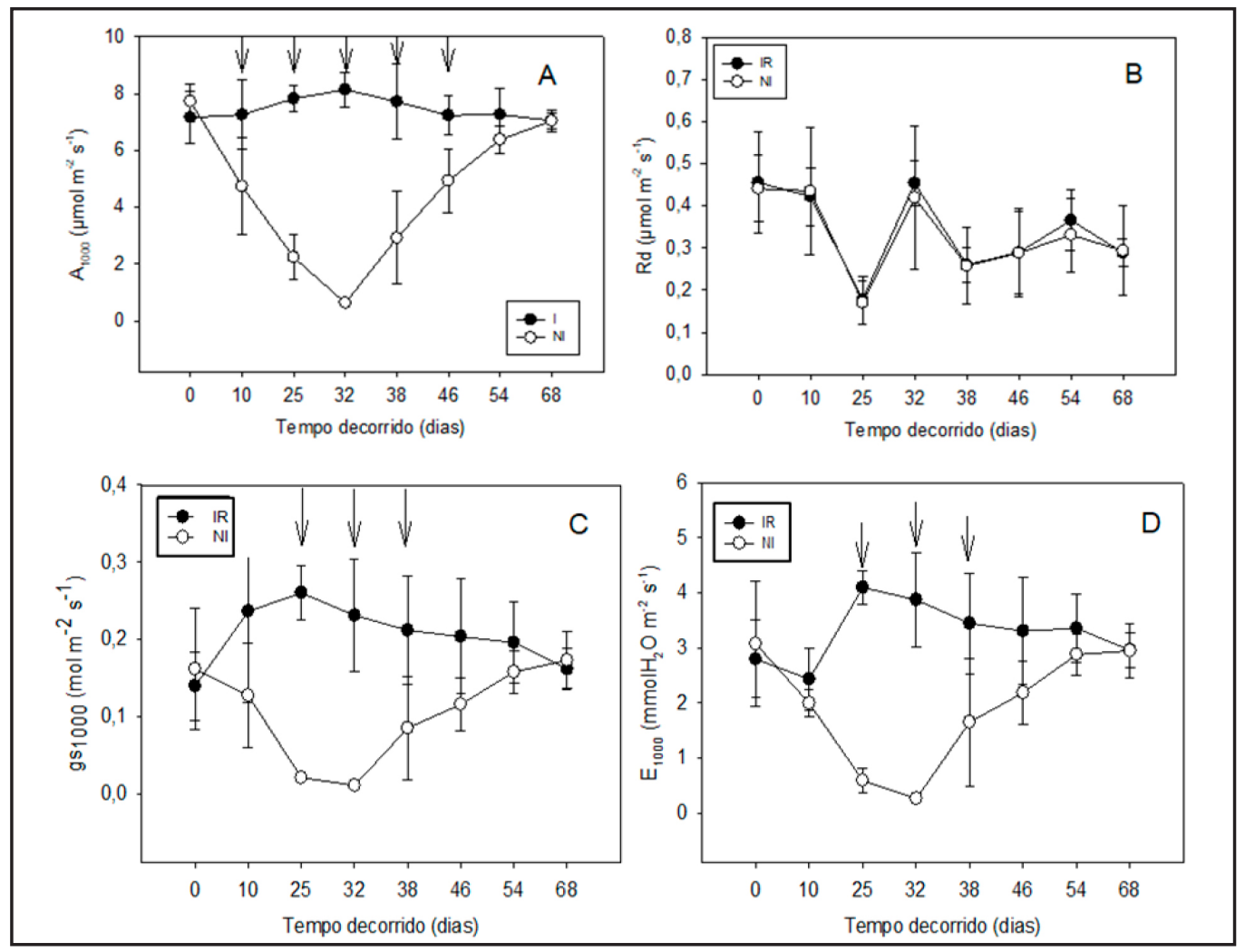

Fonte: Autores (2017)

Os valores são médias e as linhas verticais indicam os erros padrões. As setas indicam a diferença significativa entre os tratamentos pelo teste $\mathrm{t}(\mathrm{P}<0,05)$

Values are averages and vertical lines indicate standard errors. The arrows indicate the significant difference between the treatments by the test $(\mathrm{P}<0.05)$ 
A seringueira apresenta comportamento iso-hídrico, assim, espera-se que esta espécie apresente eficiente controle estomático (AYUTTHAYA et al., 2011). No presente estudo foi possível evidenciar este aspecto nas plantas jovens de Hevea brasiliensis quando se avaliou o decréscimo da condutância estomática (gs) até a fotossíntese atingir valores próximos a $0 \mu \mathrm{mol} \mathrm{m} \mathrm{m}^{-2} \mathrm{~s}^{-1}$, acompanhada da redução do potencial hídrico, no intervalo de 32 dias no tratamento NI. Além disso, a limitação estomática pode ser considerada uma estratégia da própria planta para evitar um aumento da transpiração (AYUTTHAYA et al., 2011; SEVANTO et al., 2014), e também para evitar o embolismo e cavitação no xilema (CHEN et al., 2010). Ainda com relação à respiração (rd), mesmo sendo encontrado decréscimo na terceira medida da série (ocorrência circunstancial de dias mais quentes seguidos) não houve diferença significativa entre os tratamentos ao longo de todo o período experimental. Quanto à recuperação após estresse, após o $32^{\circ}$ dia, verifica-se que ao comparar os resultados das plantas jovens de Hevea brasiliensis com os resultados de outras espécies arbóreas submetidas à abordagem e metodologia semelhantes, pode-se afirmar que a seringueira apresentou recuperação intermediária, visto que, ao ser reidratada os parâmetros de gs e $E$ foram aumentando e, após 14 dias já, havia retornado ao patamar do tratamento IR (Figura 2 C e D). Quando o indicador fisiológico analisado foi a fotossíntese, os valores entre os tratamentos IR e NI para esta variável somente foram similares após 22 dias da reidratação. Em relação à resposta mais rápida de recuperação da $g s$, confirma-se a hipótese que o gs atua de forma eficiente no controle de entrada de $\mathrm{CO}_{2}$, com o intuito de manter elevada a concentração interna de $\mathrm{CO}_{2}$ (GONÇALVES; SILVA; GUIMARÃES, 2009).

Neste trabalho não foram verificados danos oxidativos significantes nas folhas de Hevea brasiliensis quando analisados os parâmetros (razão $F_{v} / F_{m}$ e PIabs) sob deficiência hídrica (Figura $3 \mathrm{~A}$ e B). Os valores para a razão $\mathrm{F}_{\mathrm{v}} / \mathrm{F}_{\mathrm{m}}$, em termos de média geral, envolvendo todas as medidas experimentais foram da ordem de 0,84 para ambos os tratamentos (NI e IR), enquanto para a variável PIabs foi de 4,2 e 4,4 para os tratamentos (NI e IR), respectivamente. Estes resultados corroboram um estudo já realizado para outra espécie arbórea, no qual se sugere que sob estresse hídrico moderado não foram detectados decréscimos na atividade potencial do PSII em folhas de Minquartia guianensis e, por outro lado, as complicações da baixa eficiência fotoquímica sob deficiência hídrica somente seriam mais evidentes sob condições de seca mais severa, acompanhada de quase completo fechamento estomático (LIBERATO et al., 2006). Adicionalmente, estudo com espécies florestais amazônicas (Bertholletia excelsa, Carapa guianensis e Dipteryx odorata) também não demonstrou diferenças para os parâmetros de fluorescência nas folhas das espécies quando as medições foram realizadas durante diferentes períodos sazonais, de chuva e de seca, na região central da Amazônia, com ênfase nas determinações da eficiência fotoquímica do fotossistema II $\left(\mathrm{F}_{\mathrm{v}} / \mathrm{F}_{\mathrm{m}}\right)$ (MORAIS et al., 2007). Para os parâmetros da eficiência quântica máxima do fotossistema (PS) II $\left(\mathrm{F}_{\mathrm{v}} / \mathrm{F}_{\mathrm{m}}\right)$, valores em torno de 0,83 representa, em termos práticos, que a energia absorvida pelas moléculas de clorofila, nos complexos coletores de luz, foi direcionada para as etapas fotoquímicas e/ou o excesso foi dissipado na forma de calor, conforme descrito por Demmig e Björkman (1987), Neste estudo é possível ser verificado que os parâmetros da fluorescência da clorofila $a$ nas folhas de Hevea brasiliensis foram semelhantes durante todo o período de avaliação quando comparados os tratamentos NI e IR. 


\section{Figura 3 - Eficiência máxima do fotossistema II (A) e índice de desempenho (B) de plantas jovens de Hevea brasiliensis irrigadas e não irrigadas.}

Figure 3 - Maximum efficiency of photosystem II (A) and performance index (B) of young plants of Hevea brasiliensis irrigated and non-irrigated.

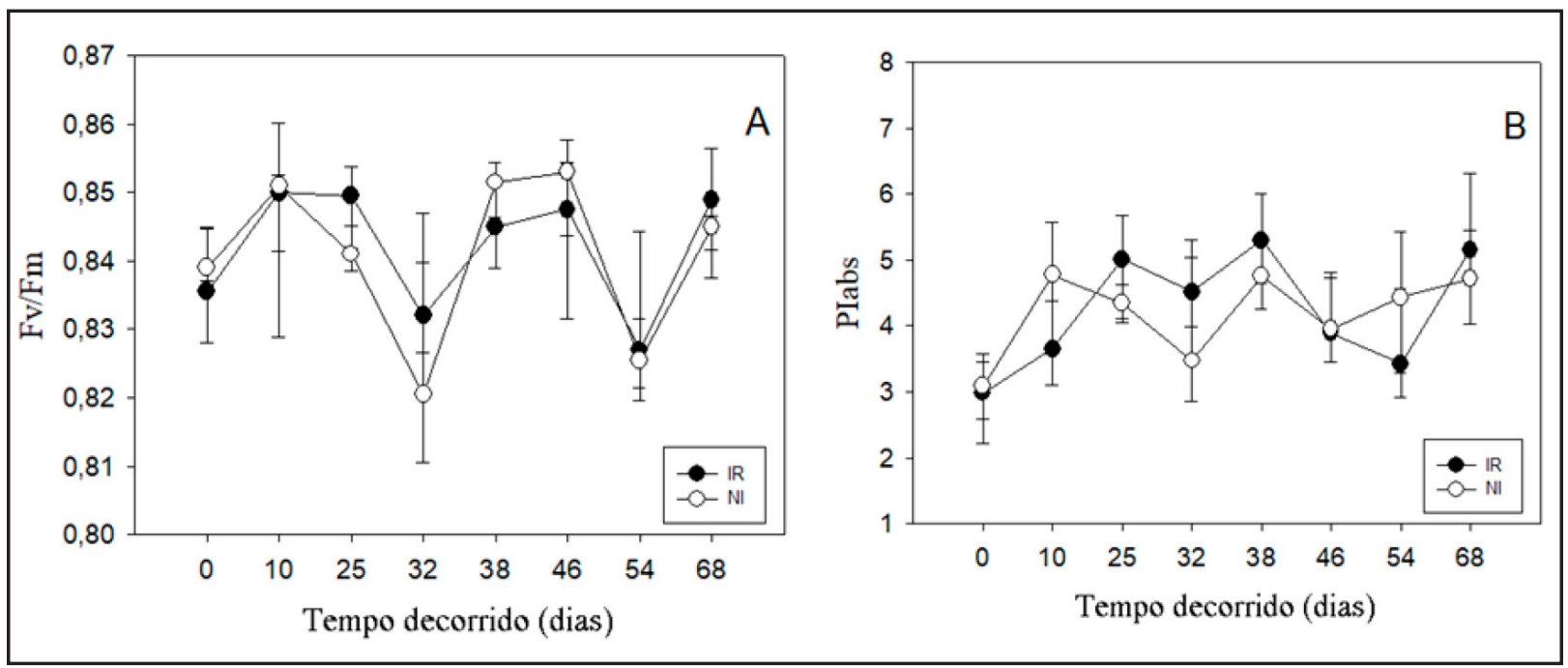

Fonte: Autores (2017)

Os valores são médias e as linhas verticais indicam os erros padrões. As setas indicam a diferença significativa entre os tratamentos pelo teste $t(P<0,05)$

The values are averages and as vertical lines indicate the standard errors. As arrows indicate the significant difference between treatments by the $t$ test $(\mathrm{P}<0.05)$

\section{Pigmentos cloroplastídicos}

No presente trabalho não foram identificadas alterações significativas nos teores de clorofila $a, b$, carotenoides, clorofila total, razão clorofila $a$ / clorofila $b$ e razão clorofila total / carotenoides em nenhum dos períodos e tratamentos (Tabela 2). Isto pode ser indicativo de que mesmo sob estresse hídrico severo, a deficiência hídrica não foi o suficiente para oxidar estes pigmentos ou inibir a síntese do mesmo, e que as proteínas associadas ao fotossistema I e II, que protegem esses pigmentos não foram desnaturadas (GALLE et al., 2009), resposta essa que pode ser confirmada ao se observarem os dados de $\mathrm{F}_{\mathrm{v}} / \mathrm{F}_{\mathrm{m}}$ e $\Phi$ PSII nos quais também não houve diferenças para estes indicadores, sugerindo estabilidade do fotossistema II para a baixa disponibilidade hídrica de folhas de seringueira. Alguns trabalhos relatam o ajustamento dos pigmentos cloroplastídicos nas plantas sob deficiência hídrica (JALLEEL et al., 2009; ANJUM et al., 2011). Isso pode ocorrer devido a eventuais danos oxidativos, porém, as plantas sintetizam antioxidantes, como exemplo os carotenoides, que favorecem a produção de enzimas como a peroxidase (JALLEEL et al., 2009). Em seringueira (clone GT1) sob deficiência hídrica ocorreram modificações nos teores de pigmentos e carotenoides, sendo essas alterações consideradas indicativo de que a integridade do cloroplasto tinha sido alterada (WANG, 2014). Entretanto, as relações diretas para estas respostas funcionais ainda precisam ser esclarecidas, uma vez que os resultados relacionam apenas com atividade de uma fração fotossistema I. 


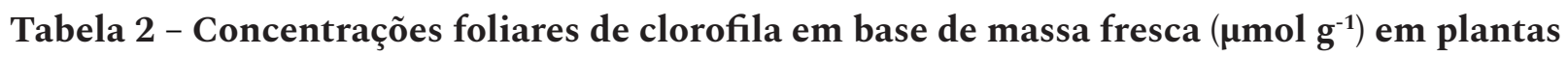
jovens de Hevea brasiliensis submetidas à deficiência hídrica e posterior recuperação.

Table 2 - Concentrations of chlorophyll on a fresh mass basis $\left(\mu \mathrm{mol} \mathrm{g}^{-1}\right)$ in young plants of Hevea brasiliensis submitted to water deficit and subsequent recovery.

\begin{tabular}{|c|c|c|c|c|}
\hline \multirow{2}{*}{ Variáveis } & \multirow{2}{*}{ Tratamentos } & \multicolumn{3}{|c|}{ Tempo (dias) } \\
\hline & & $\mathbf{0}$ & 32 & 54 \\
\hline \multirow{2}{*}{ Chl $a(\mu \mathrm{mol} / \mathrm{g})^{*}$} & IR & $2,67 \pm 0,74(\mathrm{~A})$ & $2,92 \pm 0,47(\mathrm{~A})$ & $2,02 \pm 0,59(\mathrm{~A})$ \\
\hline & $\mathrm{NI}$ & $2,87 \pm 0,36(\mathrm{~A})$ & $2,55 \pm 0,37(\mathrm{~A})$ & $2,33 \pm 0,44(\mathrm{~A})$ \\
\hline \multirow{2}{*}{$\operatorname{Chl} b(\mu \mathrm{mol} / \mathrm{g})^{*}$} & IR & $1,05 \pm 0,37(\mathrm{~A})$ & $1,18 \pm 0,23(\mathrm{~A})$ & $0,76 \pm 0,25(\mathrm{~A})$ \\
\hline & NI & $1,14 \pm 0,20(\mathrm{~A})$ & $0,95 \pm 0,22(\mathrm{~A})$ & $0,96 \pm 0,19(\mathrm{~A})$ \\
\hline \multirow{2}{*}{$\mathrm{Cx}+\mathrm{c}(\mu \mathrm{mol} / \mathrm{g})^{*}$} & IR & $1,03 \pm 0,29(\mathrm{~A})$ & $1,10 \pm 0,21(\mathrm{~A})$ & $0,74 \pm 0,22(\mathrm{~A})$ \\
\hline & NI & $1,10 \pm 0,14(\mathrm{~A})$ & $0,96 \pm 0,13(\mathrm{~A})$ & $0,84 \pm 0,17(\mathrm{~A})$ \\
\hline \multirow{2}{*}{ Chl $a /$ Chl $b^{*}$} & IR & $2,58 \pm 0,18(\mathrm{~A})$ & $2,49 \pm 0,10(\mathrm{~A})$ & $2,70 \pm 0,14(\mathrm{~A})$ \\
\hline & $\mathrm{NI}$ & $2,55 \pm 0,14(\mathrm{~A})$ & $2,87 \pm 0,51(\mathrm{~A})$ & $2,46 \pm 0,16(\mathrm{~A})$ \\
\hline \multirow{2}{*}{ Chl total $(\mu \mathrm{mol} / \mathrm{g})^{*}$} & IR & $3,72 \pm 1,10(\mathrm{~A})$ & $4,10 \pm 0,69(\mathrm{~A})$ & $3,17 \pm 0,72(\mathrm{~A})$ \\
\hline & $\mathrm{NI}$ & $4,01 \pm 0,56(\mathrm{~A})$ & $3,50 \pm 0,58(\mathrm{~A})$ & $3,29 \pm 0,63(\mathrm{~A})$ \\
\hline \multirow{2}{*}{ Chl total $/ \mathbf{C x}+\mathrm{c}^{*}$} & IR & $3,60 \pm 0,05(\mathrm{~A})$ & $3,73 \pm 0,11(\mathrm{~A})$ & $3,74 \pm 0,08(\mathrm{~A})$ \\
\hline & $\mathrm{NI}$ & $3,63 \pm 0,19(\mathrm{~A})$ & $3,61 \pm 0,21(\mathrm{~A})$ & $3,9 \pm 0,16(\mathrm{~A})$ \\
\hline
\end{tabular}

Em que: Chla $=$ Clorofila a. Chlb $=$ Clorofila $\mathrm{b} . \mathrm{Cx}+\mathrm{c}=$ Carotenoides. Chltot $=$ Clorofila total. $^{*}=$ Os valores são médias \pm desvio padrão. Letras maiúsculas distintas indicam valores significativamente diferentes entre tratamento irrigado e não irrigado pelo teste $t(P<0,05)$, a partir da terceira coluna.

\section{Açúcares solúveis totais e amido foliares}

Os resultados aqui apresentados para carboidratos foliares em plantas jovens de Hevea brasiliensis corroboram esta afirmação de que os metabólitos osmoticamente ativos estão envolvidos no mecanismo de tolerância à seca, visto que o tratamento NI aos 32 dias sem irrigação foi acompanhado de diminuição de amido, em contrapartida, houve aumento na concentração de açúcar solúvel (sacarose, glicose, frutose) (Figura 4). O consumo de amido (rafinose e sorbitol) e acúmulo ou manutenção de açúcar solúvel são importantes para fornecer carbono para a manutenção dos processos celulares e sobrevivência (MITCHELL et al., 2013), bem como para a proteger o aparato fotossintético de acúmulo espécies reativas de oxigênio (JIMÉNEZ et al., 2013). Resultado esse já observado para seringueira sob deficit hídrico, em que, além do aumento na concentração de açúcar solúvel, foi verificado o aumento de prolina (CHEN et al., 2010). Com a intensificação do deficit hídrico diversos eventos fisiológicos são induzidos, além dos já citados como fechamento estomático, redução da transpiração e menor fotossíntese (SEVANTO et al., 2014). Além disso, ocorre o ajustamento osmótico, que são necessários para manter o potencial hídrico bem como a turgescência celular em níveis adequados. Esses processos são possíveis quando ocorre o acúmulo de solutos orgânicos de baixa massa molecular no citosol, dentre eles destacam-se os açúcares solúveis, prolina e aminoácidos livres (ANJUM et al., 2011). 


\section{Figura 4 - Concentrações foliares de amido (A) e de açúcares solúveis totais (B) de plantas jovens de Hevea brasiliensis.}

Figure 4 - Leaf starch concentration (A) and soluble sugars (B) of young plants of Hevea brasiliensis.

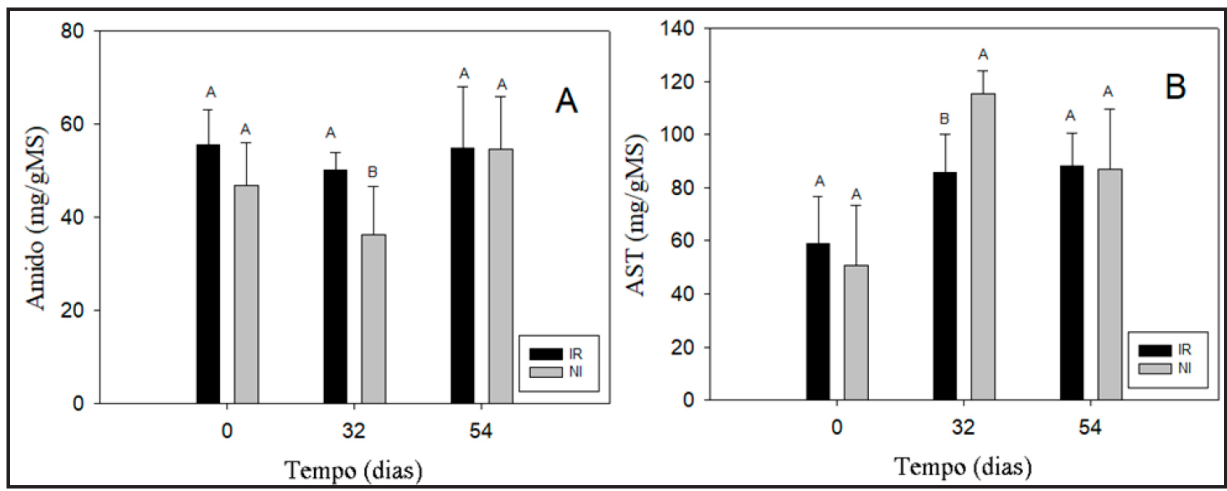

Fonte: Autores (2017)

Os valores são médias. Letras iguais indicam ausência de diferença significativa entre os tratamentos pelo teste $\mathrm{t}(\mathrm{P}<0,05)$.

Values are means. The manual can be eliminated at the same time between the tests by the $t$ test $(\mathrm{P}<0.05)$.

\section{Prolina}

O aumento de prolina também foi observado no presente estudo aos 32 dias no NI (Figura 5). O aumento da concentração da prolina está relacionada à capacidade das células de diminuir ao máximo seu $\Psi_{\mathrm{w}}$ e, no caso das células das raízes, ter a capacidade de extrair água do solo sob deficiência hídrica. Essa estratégia metabólica é considerada a primeira resposta das plantas para tentar reduzir as alterações hídricas nas células (ANJUM et al., 2011). Além disso, atua na regulação osmótica e contra os efeitos danosos produzidos por espécies reativas de oxigênio (JIMÉNEZ et al., 2013). A acumulação de prolina nas folhas em plantas sob estresse hídrico, pode estar relacionada à diminuição do potencial osmótico e aumento da eficiência no uso da água (JIMÉNEZ et al., 2013).

\section{Figura 5 - Concentrações foliares de prolina de plantas jovens de Hevea brasiliensis.}

Figure 5 - Foliar Proline Concentration of young plants of Hevea brasiliensis.

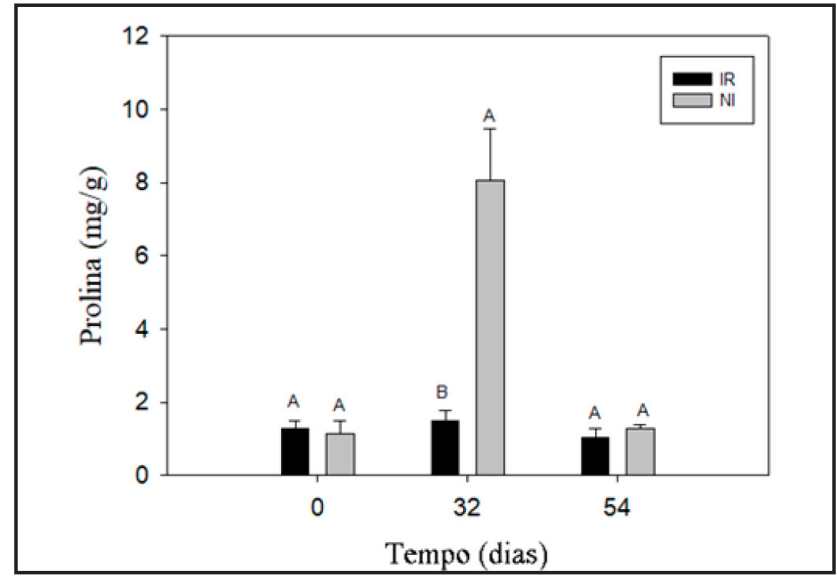

Fonte: Autores (2017)

Os valores são médias. Letras iguais indicam ausência de diferença significativa entre os tratamentos pelo teste $\mathrm{t}(\mathrm{P}<0,05)$.

Values are average. Letter equals indicate absence of significant difference between treatments by test $\mathrm{t}(\mathrm{P}<0.05)$. 


\section{Conclusão}

Os resultados confirmam a hipótese original quanto à tolerância do clone RRIM 600 (Hevea brasiliensis) ao deficit hídrico, a afirmação é sustentada pela gradativa diminuição das taxas fotossintéticas e também por não terem sido identificados sinais de fotoinibição nas mudas durante o estresse.

Para o Clone RRIM 600, a integração das variáveis da fotossíntese que não foram sensíveis ao estresse hídrico (parâmetros fotoquímicos e teores de clorofilas) sugere que o clone apresenta eficiente proteção ao aparato fotossintético em termos do fluxo de energia. A diminuição nos teores de amido e aumento nos teores de AST e de prolina durante o estresse evidencia a importância destes metabólicos para o ajustamento osmótico celular do clone sob déficit hídrico. Quanto à recuperação após o estresse hídrico, o clone RRIM 600 exibiu desempenho mediano no que se refere ao tempo de reposta, levando mais de 20 dias a partir da reidratação, não apresentando mais diferenças entre os tratamentos.

Diante do exposto, pode-se afirmar que o clone RRIM 600 ao ser submetido ao estresse hídrico suporta limitação gradual da deficiência de água, que implicará em suportar por mais tempo o deficit hídrico e suas consequências sobre processos importantes como a fotossíntese.

$\mathrm{Na}$ prática, as mudas suportariam veranicos, mas é preciso registrar que a recuperação das plantas de Hevea brasiliensis no estádio juvenil ao estresse é relativamente lenta.

\section{Agradecimentos}

Os autores desta pesquisa agradecem ao Instituto Nacional de Pesquisas da Amazônia (MCTIC-INPA) pelo suporte logístico; ao Laboratório de Fisiologia e Bioquímica Vegetal pela infraestrutura disponibilizada para realização desta pesquisa; à FAPEAM e à CAPES pelas concessões das bolsas. O Dr. José Francisco de Carvalho Gonçalves agradece ao CNPq, pela concessão da bolsa de Produtividade em pesquisa (CNPq - PQ).

\section{Referências}

ANJUM, S. A. et al. Morphological, physiological and biochemical responses of plants to drought stress. African Journal of Agricultural Research, [s.l], v. 6, n. 9, p. 2026-2032. 2011.

AYUTTHAYA, S. I. N. et al. Water loss regulation in mature Hevea brasiliensis: effects of intermittent drought in the rainy season and hydraulic regulation. Tree physiology, [s.l], v.31, n. 7, p. 751-762. 2011.

BATES, L. S.; WALDREN, R. P.; TEARE, I. D. Rapid determination of free proline for water-stress studies. Plant and soil, [s.l], v. 39, n. 1, p. 205-207. 1973.

CHEN, J. W. et al. Gas exchange and hydraulics in seedlings of Hevea brasiliensis during water stress and recovery. Tree Physiology, [s.l], v. 30, n. 7, p. 876-885. 2010.

DEMMIG, B.; BJÖRKMAN, O. Comparison of the effect of excessive light on chlorophyll fluorescence $(77 \mathrm{~K})$ and photon yield of $\mathrm{O}_{2}$ evolution in leaves of higher plants. Planta, [s.l], v. 171, n. 2, p. 171-184. 1987.

DUBOIS, M. Colorimetric method for determination of sugars and related substances. Analytical Chemistry, [s.l], v. 28, p. 350-356. 1956.

EVANS, J.; POORTER, H. Photosynthetic acclimation of plants to growth irradiance: the relative importance of specific leaf area and nitrogen partitioning in maximizing carbon gain. Plant, Cell \& Environment, [s.1], v. 24, n. 8, p. 755-767. 2001.

GALLE, A. et al. The role of mesophyll conductance during water stress and recovery in tobacco (Nicotiana sylvestris): acclimation or limitation?. Journal of Experimental Botany, [s.l], v. 60, n. 8, p. 2379-2390. 2009.

GAONA, O. D. J. C. et al. Gas exchange in young Hevea brasiliensis (Willd. Ex A. Juss.) Müll. Arg. (Euphorbiaceae) plants in Antioquia (Colombia). Corpoica Ciencia y Tecnología Agropecuaria, Mosquera, v. 19, n.1.2018.

GONÇALVES, J. F.C.; SILVA, C. E. M.; GUIMARÃES, D. G. Fotossíntese e potencial hídrico foliar de plantas jovens de andiroba submetidas à deficiência hídrica e à reidratação. Pesquisa agropecuária 
brasileira, Brasília, v. 44, n. 1, p. 8-14. 2009.

HENDRY, G. A. F.; PRICE, A. H. Stress indicators: chlorophylls and carotenoids. In: HENDRY, G. A. F.; GRIME, J. P. (Eds). Methods in Comparative Plant Ecology, London: 1993. 252 p.

JALLEEL, C. A. et al. Drought stress in plants: a review on morphological characteristics and pigments composition. International Journal of Agriculture and Biology, [s.l], v. 11, n. 1, p. 100-105. 2009.

JIMÉNEZ, S. et al. Physiological, biochemical and molecular responses in four Prunus rootstocks submitted to drought stress. Tree physiology, [s.l], v. 33, p. 1061-1075. 2013.

JIMÉNEZ-MUÑOZ, J. C. et al. Record-breaking warming and extreme drought in the Amazon rainforest during the course of El Niño 2015-2016. Scientific Reports, [s.l], v. 6. 2016.

JINAGOOL, W. et al. Clonal variability for vulnerability to cavitation and other drought-related traits in Hevea brasiliensis Müll. Arg. Journal of Plant Hydraulics, [s.l], v. 2, p. 001. 2015.

KUMAGAI, T. O. et al. How do rubber (Hevea brasiliensis) plantations behave under seasonal water stress in northeastern Thailand and central Cambodia? Agricultural and Forest Meteorology, [s.l], v. 213, p. 10-22. 2015.

KUNJET, S. et al. Effects of drought and tapping for latex production on water relations of Hevea brasiliensis trees. Kasetsart Journal: Natural Science, Bangkok, v. 47, n. 4, p. 506-515. 2013.

LAU, N. S. et al. The rubber tree genome shows expansion of gene family associated with rubber biosynthesis. Scientific reports, [s.l], v. 6. 2016

LI, Z.; FOX, J. M. Mapping rubber tree growth in mainland Southeast Asia using time-series MODIS 250 m NDVI and statistical data. Applied Geography, [s.l], v. 32, n.2, p. 420-432. 2012.

LIBERATO, M. A. R. et al. Leaf water potential, gas exchange and chlorophyll a fluorescence in acariquara seedlings (Minquartia guianensis Aubl.) under water stress and recovery. Brazilian Journal of Plant Physiology, [s.l], v. 18, n. 2, p. 315-323. 2006.

LICHTENTHALER, H.K.; WELLBURN, A.R. Determination of total carotenoids and chlorophyll $a$ and $b$ of leaf extracts in different solvents. Biochemical Societ Transactions, [s.l], p. 591-603. 1983.

MELO, L. A. et al. Estaquia e efeito da deficiência hídrica ou inundação sobre características morfoanatômicas de Cestrum axillare Vell. Ciência Florestal, Santa Maria, v. 27, n. 1, p. 325-337. 2017.

MITCHELL, P. J. et al. Drought response strategies define the relative contributions of hydraulic dysfunction and carbohydrate depletion during tree mortality. New Phytologist, [s.l], v. 197, n. 3, p. 862872. 2013.

MORAIS, R. R. D. et al. Chloroplastid pigment contents and chlorophyll a fluorescence in amazonian tropical three species. Revista árvore, Viçosa, v. 31, n. 5, p. 959-966. 2007.

PRIYADARSHAN, P. M. et al. Yielding potential of rubber (Hevea brasiliensis) in sub-optimal environments. Journal of crop improvement, [s.l], v. 14, n. 2, p. 221-247. 2005.

SANTOS JUNIOR, U. M.;GONÇALVES,J.F.C.; FELDPAUSCH,T.R. Growth, leaf nutrient concentration and photosynthetic nutrient use efficiency in tropical tree species planted in degraded areas in central Amazonia. Forest Ecology and Management, [s.l], v. 226, n. 1, p. 299-309. 2006.

SCHOLANDER, P. F. et al. Hydrostatic pressure and osmotic potential in leaves of mangroves and some other plants. Proceedings of the National Academy of Sciences, [s.l], v.52, n. 1, p. 119-125. 1964.

SEVANTO, S. et al. How do trees die? A test of the hydraulic failure and carbon starvation hypotheses. Plant, Cell \& Environment, [s.l], v. 37, n. 1, p. 153-161. 2014.

STRASSER, R. J. et al. Simultaneous in vivo recording of prompt and delayed fluorescence and 820-nm reflection changes during drying and after rehydration of the resurrection plant Haberlea rhodopensis. Biochimica et Biophysica Acta, [s.l], v. 1797, n. 6, p. 1313-1326. 2010.

WANG, L. F. Physiological and molecular responses to drought stress in rubber tree (Hevea brasiliensis Muell. Arg.). Plant Physiology and Biochemistry, [s.l], v. 83, p. 243-249. 2014. 